\title{
Spatiotemporal Stability of a Femtosecond Hard-X-Ray Undulator Source Studied by Control of Coherent Optical Phonons
}

\author{
P. Beaud, ${ }^{*}$ S. L. Johnson, A. Streun, R. Abela, D. Abramsohn, D. Grolimund, F. Krasniqi, \\ T. Schmidt, V. Schlott, and G. Ingold \\ Paul Scherrer Institut, CH-5232 Villigen PSI, Switzerland \\ (Received 1 June 2007; published 26 October 2007)
}

\begin{abstract}
We report on the temporal and spatial stability of the first tunable femtosecond undulator hard-x-ray source for ultrafast diffraction and absorption experiments. The 2.5-1 $\AA$ output radiation is driven by an initial $50 \mathrm{fs}$ laser pulse employing the laser-electron slicing technique. By using x-ray diffraction to probe laser-induced coherent optical phonons in bulk bismuth, we estimate an x-ray pulse duration of $140 \pm$ $30 \mathrm{fs}$ FWHM with timing drifts below $30 \mathrm{fs}$ rms measured over 5 days. Optical control of coherent lattice motion is demonstrated.
\end{abstract}

Pump-probe diffraction and absorption experiments with $\mathrm{x}$ rays have the potential to capture transient structures during chemical and physical reactions on the fundamental time scales of atomic motion. At future free-electron-laser (XFEL) facilities laser pump, $\mathrm{x}$-ray probe experiments will be an important class of time-dependent experiments [1$3]$. Accurate knowledge of pulse lengths, relative time delay, and the relative spatial position between the pump and probe beams are critical for successful pump-probe experiments. Although there is considerable experience on these issues with laser-based sources of pulsed light, accelerator-based sources offer new challenges. Demonstrated performance of such sources is scarce but needed for the design of future XFEL experiments.

Employing an electro-optical technique, single-shot measurements of the difference in arrival time with a short term accuracy of $60 \mathrm{fs}$ rms has recently been demonstrated at the Sub-Picosecond Pulse Source [4] allowing postsorting of time-stamped laser-x-ray pump-probe data [5]. In this case, multishot data acquisition was done within $20 \mathrm{mi}-$ nutes. Nonetheless, anticipating a XFEL pulse length below $10 \mathrm{fs}$, the timing control and resolution required may be achieved only by combining active synchronization and single-shot time-stamping measurements. There is an analogous situation regarding long-term spatial and temporal stability at spontaneous, low intensity femtosecond $\mathrm{x}$-ray sources where pump-probe data must be accumulated stroboscopically over millions of shots. To evaluate the limits of active stabilization, we have done such an experiment using a tunable femtosecond undulator $\mathrm{x}$-ray source (Fig. 1) recently installed at the Swiss Light Source (SLS) [6], a storage ring with a highly stable electron beam operated in top-up mode and controlled by a fast orbit feedback system. Femtosecond $\mathrm{x}$ rays are generated using slice-energy modulation [7-11]. Because both the optical pump and the x-ray probe pulses are derived from the same Ti:sapphire laser, they are inherently synchronized.

Femtosecond pulses are initially created in a conventional Kerr-lens mode-locked oscillator phase-locked to the fifth submultiple of the synchrotron rf-master oscillator $(500 \mathrm{MHz})$ with an estimated jitter $<1 \mathrm{ps}$. These pulses are split into two parallel optical paths: one for exciting a sample optically (the "pump" branch) and another for modulating the energy of electrons in the storage ring (the "probe" branch). The pump pulses enter a regenerative amplifier ( $115 \mathrm{fs}$ FWHM, $1 \mathrm{kHz}, 2.1 \mathrm{~mJ}, 800 \mathrm{~nm}$ ) and are transported to the experiment via a $22 \mathrm{~m}$ long vacuum pipe. The probe pulses are amplified in two steps: first by a regenerative amplifier and then by a symmetrically pumped 2-pass amplifier (50 fs FWHM, $1 \mathrm{kHz}, 5.2 \mathrm{~mJ}$, $805 \mathrm{~nm})$. A nearly diffraction-limited spatial profile $\left(M^{2} \leq\right.$ 1.4) for efficient slicing is ensured by cryogenic cooling of the 2-pass amplifier laser crystal. The intense femtosecond laser pulses are focused in vacuum over an optical distance of $45 \mathrm{~m}$ to interact with the electrons at the modulator with a Rayleigh length of $0.6 \mathrm{~m}$ (beam waist $w_{0}=490 \mu \mathrm{m}$ ). The environmental temperature is stabilized to $<1^{\circ} \mathrm{C}$.

To minimize bunch lengthening, the storage ring installations for short pulse generation are placed in a single $11 \mathrm{~m}$ long straight section. The associated breaking of the ring periodicity imposed constraints on the electron beam parameters in order to avoid deterioration of the storage ring dynamic aperture [12]. The slicing spectrometer consists of a modulator (wiggler: $B_{w, \text { eff }}=1.98 \mathrm{~T}, g=$ $11 \mathrm{~mm}, \quad \lambda_{w}=138 \mathrm{~mm}, N_{w}=17$ periods) for energy modulation, chicane dipoles for pulse separation, quadrupole-focusing magnets to reduce the beam height, and a radiator [13] (in-vacuum undulator: $B_{u \text {,eff }}=0.92 \mathrm{~T}$, $g=5 \mathrm{~mm}, \lambda_{u}=19 \mathrm{~mm}, N_{u}=96$ periods) operated at high harmonics providing a monochromatic $\mathrm{Si}(111)$ flux of $8 \times 10^{12}$ photons/s in a $0.014 \%$ bandwidth (aperture $240 \times 54 \mu \mathrm{rad}^{2}$, average current $400 \mathrm{~mA}$ ). When the modulator magnetic field $B_{w, \text { eff }}$ is tuned to resonance $\lambda_{L}=$ $\lambda_{w}\left(1+K_{\mathrm{eff}}^{2} / 2\right) / 2 \gamma^{2} \quad$ (laser wavelength $\lambda_{L}=805 \mathrm{~nm}$, deflection parameter $K_{\text {eff }}=0.934 B_{w, \text { eff }}[\mathrm{T}] \quad \lambda_{w}[\mathrm{~cm}]$, Lorentz factor $\gamma=4720$ ) by changing the wiggler gap, the laser induces an energy modulation in the copropagating electron bunch. Optimal energy transfer is achieved if 


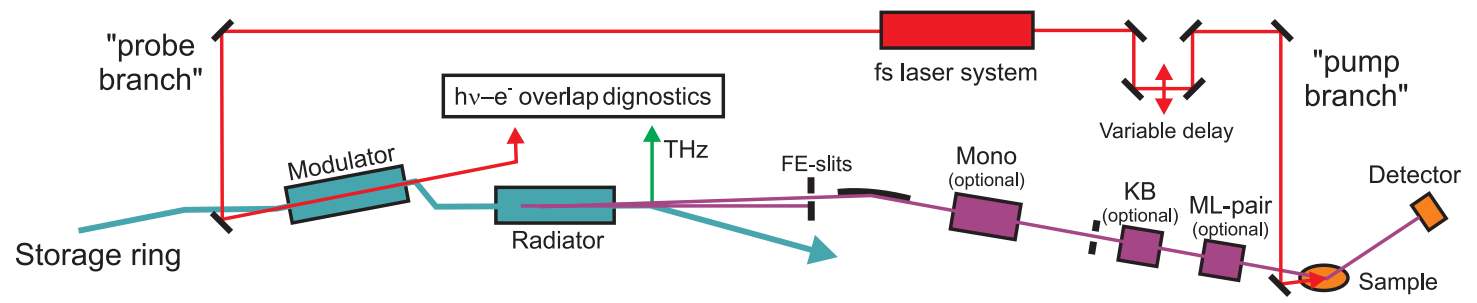

FIG. 1 (color). Layout of the laser-X-ray pump-probe experiment at the $\mu$ XAS beam line at the SLS.

the number of modulator periods $N_{w}$ matches the number of laser optical cycles $\left(N_{L}=18\right)$. The chicane induces a spatial $( \pm 2 \mathrm{~mm})$ and angular $( \pm 0.5 \mathrm{mrad})$ horizontal separation of the sliced beam at the radiator center. Movable apertures in the x-ray beam line at $12 \mathrm{~m}$ distance from the radiator block radiation from the core beam while allowing $\mathrm{x}$ rays emitted by the satellite beam to pass. For sufficient suppression of the core beam background, only radiation from electrons with positive energy modulation $\Delta E / E>$ $5 \times 10^{-3} \simeq 6 \sigma_{\Delta E / E}$ is accepted (where $\sigma_{\Delta E / E}$ is the natural energy spread, $E=2.41 \mathrm{GeV}$ ). According to simulations, a laser pulse energy of $2 \mathrm{~mJ}$ provides a maximum energy modulation of $\sim 1 \%(24 \mathrm{MeV})$.

As shown in Fig. 2, slicing operation is optimized and monitored online by measuring the intensity of coherent radiation emitted by the modulated electron bunches in the terahertz range [14] at a diagnostic port installed at the first bending magnet after the interaction straight. The quadratic dependence of the terahertz radiation on the number of modulated electrons gives sufficient dynamic range to optimize the spatial and spectral laser-electron overlap. The spectral response $\left(\leq 33 \mathrm{~cm}^{-1}\right)$ of the He-cooled $(4.2 \mathrm{~K})$ InSb-bolometer records the long-wavelength co-
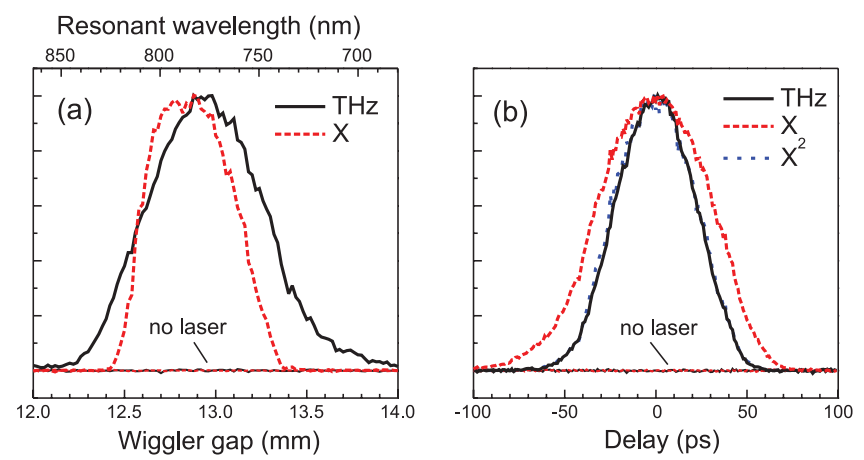

FIG. 2 (color). Tuning of the laser-electron interaction by monitoring coherent far-IR radiation and incoherent sliced $\mathrm{x}$ ray flux. (a) For the sliced $x$ rays, the resonant wavelength is redshifted because only radiation from a subset of electrons with $0.6 \%-0.8 \%$ energy gain is accepted by the first mirror in the beam line. (b) Optimization of the temporal overlap between the laser pulses and the isolated electron bunch. As expected, a linear (square) dependence on the number of sliced electrons is observed for the incoherent (coherent) radiation channel. A pulse width of 85 ps FWHM is measured for the isolated electron bunch $(4 \mathrm{~mA})$. herent radiation emitted during the first turn of the modulated electrons around the storage ring.

The femtosecond photon flux measured with an avalanche photodiode is $2 \times 10^{5}\left(6 \times 10^{4}\right)$ photons/s in a $0.1 \%$ bandwidth at $5(8) \mathrm{keV}$, for $2 \mathrm{~mJ}$ slicing laser energy and $4 \mathrm{~mA}$ isolated single bunch current corresponding to a peak current of $60 \mathrm{~A}$ [12]. The stability of relevant parameters for the laser, electron, and x-ray beams recorded during extended pump-probe scans is shown in Fig. 3. Because of photon absorbers installed along the spectrometer and adjustable slits in the beam line, $\mathrm{x}$ rays emitted by the 85 ps FWHM core beam are suppressed by $10^{3}: 1$. The background is instead limited by the "halo" of incompletely damped previously modulated electrons [11] that limits the signal to background ratio to $\sim 50: 1$.

To characterize the $\mathrm{x}$-ray pulse length and timing stability, we use x-ray diffraction to measure high-amplitude phonon dynamics of photoexcited bismuth [5,15]. Strong optical excitation of the near-surface region of the crystal results in large amplitude coherent $A_{1 g}$ optical phonons, corresponding to motion of atoms along the body diagonal of the rhombohedral unit cell. Since this motion alters the structure factor, we can observe the coherent phonon dynamics by measuring the diffracted intensity from certain Bragg reflections as a function of time. Specifically, we
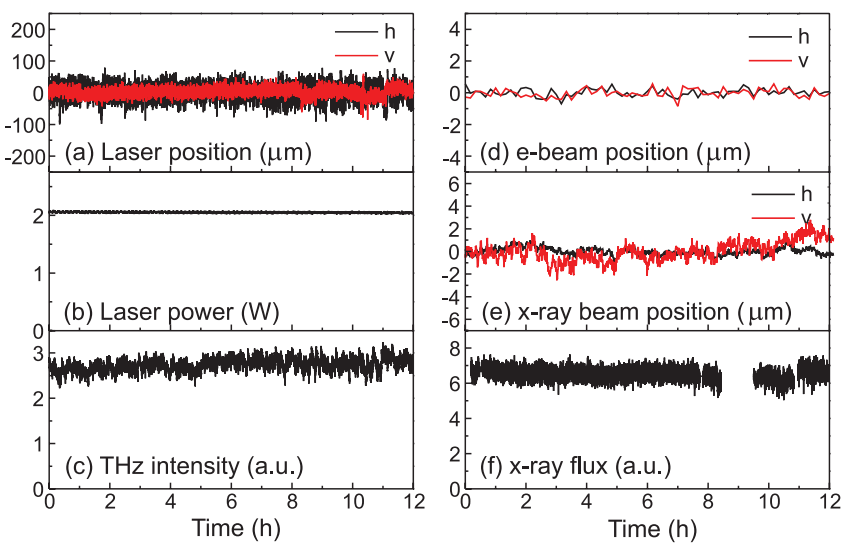

FIG. 3 (color). Slicing diagnostic chart recorded online over 12 hours. Monitored are (a) the laser position stability at the interaction point controlled by position feedback, (b) the laser power, (c) the terahertz intensity, (d) the electron beam position at the radiator (BPM readings), (e) the x-ray beam position (XBPMs $8.6 \mathrm{~m}$ downstream of radiator, sampling $0.1 \mathrm{~Hz}$ ), and (f) the femtosecond $\mathrm{X}$-ray intensity. 
have used grazing-incidence x-ray diffraction on the (210) and (111) Bragg reflections of single crystal bulk bismuth samples to observe this phenomenon. The $\mathrm{x}$-ray energy was set to $7.15 \mathrm{keV}$ and the angle of incidence to approximately $0.5^{\circ}$, limiting the probe depth to $<15 \mathrm{~nm}$ from photoabsorption in the crystal. A toroidal Rh-coated mirror with a grazing incidence of 3 mrad collimates the $\mathrm{x}$ rays vertically and creates a weak horizontal focus on the sample. Close to the sample, a single Kirkpatrick-Baez (KB) mirror focuses the beam vertically to a size of $10 \mu \mathrm{m}$. The sample is aligned so that the plane of incidence is vertical, resulting in a spot size on the sample of $300 \mu \mathrm{m} \times 1.1 \mathrm{~mm}$. The pump laser is incident on the sample at a grazing angle of $\sim 14^{\circ}$, resulting in a geometrical loss in time resolution of $110 \pm 20 \mathrm{fs}$ FWHM. The probe signal is sampled with twice the pump frequency to record alternately pumped and unpumped diffraction data to compensate for slow time-scale drifts in the source, pump laser, detector, or electronics [16]. The laser fluence of $0.5-2.5 \mathrm{~mJ} / \mathrm{cm}^{2}$ on the sample allowed measurements for many hours without visible crystal damage at a given spot. Depending on the use of a single- or doublemultilayer monochromator and losses due to the KB mirror and air absorption, the incident flux on the sample was $(2-8) \times 10^{5}$ photons $/ \mathrm{s}$ in a $1.2 \%$ bandwidth.

Time-delay scans over the range 3-4 ps with step size 30-60 fs lasted 1.5-7.5 hours depending on crystal quality (mosaicity) and laser fluence. In Fig. 4(a), we show data accumulated on the (210) reflection for 7.5 hours yielding an effective time resolution of $195 \pm 25$ fs FWHM including time-delay drifts. The scan of the more efficient (111) diffraction at a higher fluence of $2.24 \mathrm{~mJ} / \mathrm{cm}^{2}$ in Fig. 4(b) is integrated over only 90 minutes. Fitting these data to a simple model of a displacive excitation [17], we obtain an average phonon frequency of $2.60 \pm 0.05$ and $2.46 \pm$ $0.02 \mathrm{THz}$, respectively, which is comparable to the fluence dependence measured on bismuth thin films [5]. Deconvolving the fit result with the duration of the pump pulse and the noncollinear geometry yields an effective x-ray pulse length of $140 \pm 30 \mathrm{fs}$ FWHM. The timing drifts are below 30 fs rms over 5 days [see Fig. 4(c)].

To further demonstrate the spatial and temporal stability of the pump and probe beams, we used a sequence of two pulses to control the population of the $A_{1 g}$ phonon mode. In this double-pump experiment, the amplitude of the coherent atomic motion can be manipulated [18] through the delay of the second pulse by keeping the electronic excitation constant [19]. The spectra shown in Fig. 5 are obtained by delaying the second pump pulse over one oscillation period of the coherent optical phonon. Its amplitude is either amplified [Figs. 5(b) and 5(f)] or canceled [Fig. 5(d)], depending on the relative phase of the second pulse with respect to the coherent phonon generated by the first pulse. The simulated curves are obtained by assuming a linear dependence on laser fluence to calculate the fractional electron-hole pair density $n(t)$ (as a fraction of the
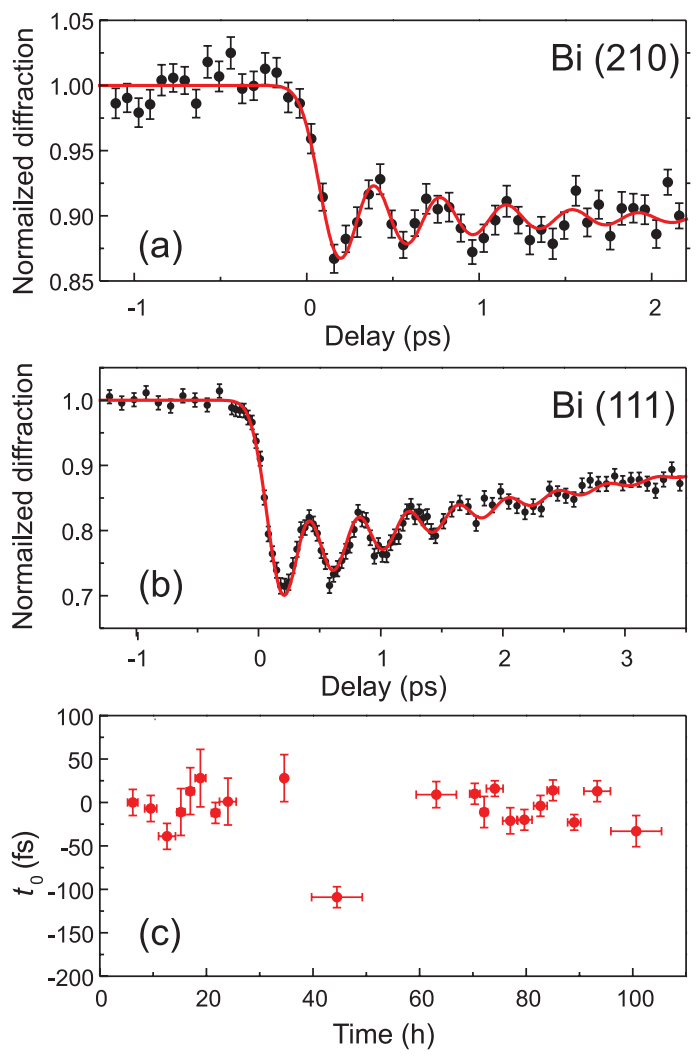

FIG. 4 (color). Normalized integrated diffracted intensity from the bulk (a) $\mathrm{Bi}(210)$ and (b) $\mathrm{Bi}$ (111) reflection at $7.15 \mathrm{keV}$ as a function of pump-probe delay for excitation fluences of 1.53 and $2.24 \mathrm{~mJ} / \mathrm{cm}^{2}$, respectively. The lines are fits to the data. (c) Measured time drifts during 5 days of consecutive scans. The horizontal error bars indicate the length of the scans.

total number of valence electrons) by solving:

$$
\dot{n}=n_{1} P(t)+n_{2} P(t+d t)-k_{n} n(t),
$$

where $P(t)$ is the normalized Gaussian shape of the pump pulses $\left[\int P(t) d t=1\right]$ and $n_{1} \approx n_{2}$ are the fractional electron-hole pair densities created by the first and second laser pulses separated in time by $\Delta t$. The rate $k_{n}=$ $0.22 \mathrm{ps}^{-1}$ accounts for the decay of the electronic excitation. The atomic motion $z(t)$ along the (111) direction (in units of the length $c=1.18 \mathrm{~nm}$ of the hexagonal unit cell) is then calculated with

$$
\ddot{z}=-\frac{1}{c^{2} m} \frac{d}{d z} V[n(t), z]-k_{z} \dot{z},
$$

where $m$ is the bismuth atom mass and $V[n(t), z]$ is the atomic potential energy of Ref. [19]. The rate $k_{z}=$ $1.8 \mathrm{ps}^{-1}$ is introduced here to describe the observed damping of the coherent oscillation. The normalized timedependent diffraction signal is finally calculated [5]:

$$
I(t) / I(0)=\cos ^{2}[6 \pi z(t)] / \cos ^{2}[6 \pi z(0)],
$$

$2 z(0)=0.46719$ being the equilibrium distance of the two basis atoms along the trigonal axis. Equation (3) is valid only in the limit of a thin film, which for our experimental 


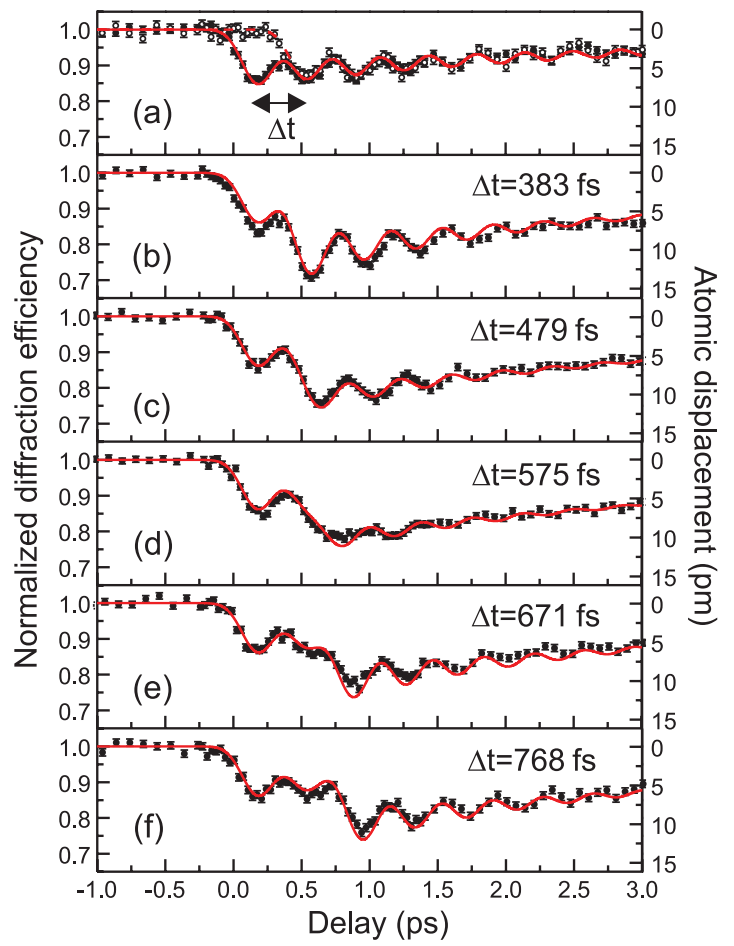

FIG. 5 (color). Measured (symbols) and simulated (solid curves) bismuth (111) coherent optical phonon oscillation controlled by a 2-pulse excitation scheme. The absorbed energy density per pulse was $1.12 \mathrm{~mJ} / \mathrm{cm}^{2}$. Right axis: Atomic displacement as inferred from the simulation.

conditions is a valid approximation (applying the full dynamical diffraction theory [20] led to negligible corrections on the order of $0.5 \%$ ). The absorption depth of the laser is $\approx 17 \mathrm{~nm}$ [21], and the probe depth of the $\mathrm{x}$ rays is $15 \mathrm{~nm}$. Assuming that each absorbed photon creates an electron-hole pair, the initial carrier density in the probed volume should be $1.76 \times 10^{21} \mathrm{~cm}^{-3}$ at an absorbed fluence of $1.12 \mathrm{~mJ} / \mathrm{cm}^{2}$ corresponding to $n_{1}=1.23 \%$ [19]. However, fitting the single pulse excitation data shown in Fig. 5(a) with Eqs. (1)-(3) yields significantly lower values of $n_{1}=0.63 \%$ and $n_{2}=0.59 \%$. This discrepancy might be explained by excited carrier absorption and fast hot carrier diffusion. Without any further adjustment (besides a small shift in time zero $t_{0}$ of 10-40 fs), the simulated transients are compared to the experimental data in Figs. 5(b)-5(f), showing good agreement with the observed dynamics.

We have presented the performance of a $140 \pm 30 \mathrm{fs}$ FWHM undulator source in the angstrom range for laser pump, $\mathrm{x}$-ray probe experiments under optimum conditions in terms of spatial stability of the laser, electron, and x-ray beams. Inherent synchronization enables multishot data accumulation for days with timing stability of $30 \mathrm{fs}$ rms and instrumental time resolution of $85 \mathrm{fs}$ rms. A crucial test is the successful demonstration of coherent control of real space atomic motion at such time scales. To our knowl- edge, this is the first optical control experiment where the coherent atomic structural response has directly been measured in real space with hard $\mathrm{x}$ rays on the $100 \mathrm{fs}$ time scale. Laser manipulation of electron bunches most likely will play an important role at future XFEL facilities to generate $\leq 10 \mathrm{fs}$ x-ray pulses in a controlled way $[22,23]$. The performance reported in this Letter provides a starting point to design XFEL pump-probe experiments on these short time scales.

We acknowledge the assistance of $K$. Holldack, J. Bahrdt, and A. Gaupp (BESSY), V. Vobly (BINP), and O. Chubar (SOLEIL). We thank R.W. Schoenlein, A. Zholents (LBL), and S. Khan (University of Hamburg) for fruitful discussions. We are indebted to W. Bulgheroni, Q. Chen, A. Keller, and L. Schulz of the PSI technical staff. This project has been supported by special funds provided by the ETH board.

*paul.beaud@psi.ch

[1] M. Altarelli et al., DESY Report No. 2006-097, 2006.

[2] J. Arthur et al., SLAC Report No. SLAC-R-521, 1998.

[3] SCSS X-FEL Conceptual Design Report, RIKEN, Harima Institute, Sayo, Japan, 2005.

[4] A. L. Cavalieri et al., Phys. Rev. Lett. 94, 114801 (2005).

[5] D. M. Fritz et al., Science 315, 633 (2007).

[6] G. Ingold et al., in Ninth International Conference on Synchrotron Radiation Instrumentation, edited by J.-Y. Choi and S. Rah, AIP Conf. Proc. No. 879 (AIP, New York, 2006), p. 1198.

[7] L. H. Yu, Phys. Rev. A 44, 5178 (1991).

[8] A. A. Zholents and M. S. Zolotorev, Phys. Rev. Lett. 76, 912 (1996).

[9] R. W. Schoenlein et al., Science 287, 2237 (2000).

[10] R. W. Schoenlein et al., Appl. Phys. B 71, 1 (2000).

[11] S. Khan et al., Phys. Rev. Lett. 97, 074801 (2006).

[12] A. Streun et al., in Proceedings of the European Particle Accelerator Conference, Edinburgh, 2006 (http://www. jacow.org, 2006), p. 3427.

[13] G. Ingold et al., in Ninth International Conference on Synchrotron Radiation Instrumentation, edited by J.-Y. Choi and S. Rah, AIP Conf. Proc. No. 879 (AIP, New York, 2006), p. 388.

[14] K. Holldack et al., Phys. Rev. Lett. 96, 054801 (2006).

[15] K. Sokolowski-Tinten et al., Nature (London) 422, 287 (2003).

[16] M. Saes et al., Rev. Sci. Instrum. 75, 24 (2004).

[17] H. J. Zeiger et al., Phys. Rev. B 45, 768 (1992).

[18] M. Hase et al., Appl. Phys. Lett. 69, 2474 (1996).

[19] E. D. Murray et al., Phys. Rev. B 72, 060301 (2005).

[20] A. M. Afanas'ev and I. P. Perstnev, Acta Crystallogr. Sect. A 25, 520 (1969).

[21] H.-J. Hagemann, W. Gudat, and C. Kunz, J. Opt. Soc. Am. 65, 742 (1975).

[22] A. A. Zholents and G. Penn, Phys. Rev. ST Accel. Beams 8, 050704 (2005).

[23] E. L. Saldin, E. A. Schneidmiller, and M. V. Yurkov, Phys. Rev. ST Accel. Beams 9, 050702 (2006). 